\title{
ALGUNOS MICROHONGOS GEOFILICOS DE LAS PLANICIES SEMIARIDAS DEL NOROESTE DE LA PROVINCIA DE SAN LUIS (ARGENTINA)
}

(Some geophilic microfungi from Norwest semiarid plains in the province of San Luis, Argentine)

\author{
Magdalena Mangiaterra*, Gustavo Giusiano*, Iliana González* \\ * Departamento Micología. Instituto de Medicina Regional. \\ Universidad Nacional del Nordeste. Av. Las Heras 727, 350 \\ Resistencia, Argentina. E-mail: magmangi@bib.unne.edu.ar
}

Palabras claves: Microhongos geofílicos, suelos semiáridos, Argentina.

Key words: Geophilic microfungi, semiarid soils, Argentine.

\section{RESUMEN}

Por las técnicas del anzuelo queratínico y de dilución, se estudiaron tierras de las planicies semiáridas del noroeste de la provincia de San Luis (Argentina), en invierno y verano. Con la técnica del anzuelo queratínico se aislaron 12 géneros y 15 especies fúngicas, mientras en las procesadas por dilución en PDA, 15 géneros y 29 especies. La mayoría de las detectadas con la primera técnica pertenecieron a los Onygenales (65,4\%), siendo Chrysosporium indicum el de más alta frecuencia, mientras que en las diluciones, la mayoría correspondió a hongos mitospóricos (>75\%), dominando los integrantes del género Aspergillus $(35,8 \%)$. Con ambas técnicas la riqueza de especies fue levemente superior en verano y aún cuando la diversidad general fue baja, se aprecia la capacidad de adaptación y sobrevivencia de ciertos géneros y especies en ambientes edáficos adversos.

\section{INTRODUCCION}

Después de los insectos, los hongos constituyen el grupo que incluye mayor número de individuos. Su inusual y amplia variedad morfológica se une a un singular y diverso patrón de comportamiento y las diferentes estrategias de vida que han desarrollado, explican su enorme importancia en la evolución, en el ecosistema, en el progreso humano, y en la mayoría de los procesos que tienen lugar en la Tierra (1).

La actividad de los hongos en el suelo es importante por el funcionamiento de los ciclos biogeoquímicos,
ABSTRACT

Soils from northwestern semiarid plains in the province of San Luis (Argentina) were examined by means of keratinic bait and dilution techniques both in winter and summer. The first technique yielded 12 genera and 15 fungal species whereas those processed in PDA dilution resulted in 15 genera and 29 species. Most fungi detected with the former technique belonged to Onygenales (65.4\%), being Chrysosporium indicum the most frequently occurring, while in dilutions mitosporic fungi $(<75 \%)$ showed the highest occurrence, those of the genus Aspergillus having dominance (35.8\%). The richness of species was fairly higher with both techniques and even though general diversity was low, the ability of certain genera and species to adapt and survive under adverse edaphic environments could be clearly seen.

especialmente en el ciclo del carbono. En Argentina las particulares características geográficas y climáticas determinan la existencia de una interesante micota sobre la cual existe limitado conocimiento, tanto a nivel básico como aplicado, debido a la presencia de especies de gran relevancia en los ámbitos de la patología humana, forestal y agrícola.

La Provincia de San Luis está ubicada en el centro geográfico de la República Argentina, a 800 Km del Océano Atlántico y a $700 \mathrm{Km}$ del puerto chileno de Valparaíso en las costas del océano Pacífico. Por su latitud, se halla ubicada dentro de la zona templada, pero su continentalidad imprime al clima ciertos rasgos típicos, acentuados 


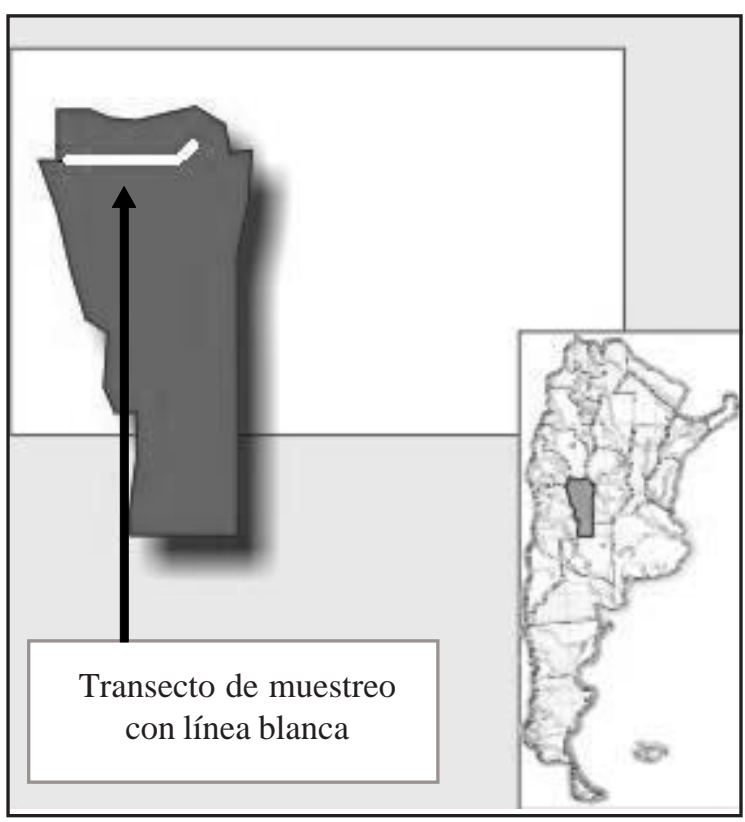

Figura1. Transecto de muestreo y posición geográfica

por el relieve. La temperatura promedio anual es de $17^{\circ} \mathrm{C}$. La precipitación media anual alcanza los $400 \mathrm{~mm}$ y disminuye gradualmente de Este a Oeste. Esta provincia se divide en tres regiones con distinto tipo de clima. La zona norte, donde se recolectaron las muestras, es la más árida de la provincia. Ello se debe a la influencia de los vientos provenientes del cuadrante norte, continental, cálido y seco; las escasas precipitaciones inferiores a 200 $\mathrm{mm}$ anuales se concentran en verano, cayendo como fuertes chaparrones que provocan intensa denudación (2).

El presente trabajo se efectuó con el fin de conocer la ocurrencia natural y la riqueza de especies de geohongos queratinofílicos y no queratinofílicos en dos períodos estacionales en un área con características climáticas adversas.

\section{MATERIALES Y METODOS}

Las muestras se recolectaron en el sector norte de la provincia de San Luis, a lo largo de la ruta provincial $\mathrm{N}^{\circ}$ 20 que recorre una amplia franja de transición entre el Monte y el Chaco, unidades biogeográficas caracterizadas por una vegetación de matorrales y por un bosque seco caducifolio, respectivamente (Fig.1).

En la zona norte de la provincia, la orogenia fracturó en bloques la antigua peniplanicie, lo que determinó el perfil asimétrico con pendiente suave y regular hacia el este y la ladera más abrupta hacia el oeste. La planicie septentrional es una cuenca sedimentaria en la que mueren los escasos ríos de la vertiente de la sierra, en la que por su aridez, el pozo, el balde o la represa son el factor decisivo de la posibilidad de radicación de población (2). Los bloques escalonados de oeste a este están coronados de pampas que se hallan, la superior, entre los 1500 y 1000 m.s.n.m. y la intermedia o peniplanicie entre los 600 y $100 \mathrm{~m}$.

\section{Muestreo}

El muestreo se realizó a lo largo de los $200 \mathrm{Km}$ que separan la población de Quines y el punto límite de la provincia ( $31^{\circ} 52^{\prime}$ 'S y $67^{\circ} 20^{\prime} \mathrm{O}$ ), entre los 500 m.s.n.m. y la Sierra de las Quijadas (1200 m). Las muestras se tomaron cada 18-20 Km, alejándose entre 200 y 300 m del camino principal. Las 20 muestras obtenidas fueron: 10 en verano (Enero) y 10 en invierno (Junio). Los lugares de muestreo en verano, se marcaron en el terreno ya sea con estacas o pintura sobre zonas rocosas cercanas, para repetir en puntos semejantes el muestreo de invierno.

Las muestras individuales en cada sector se tomaron de la capa superficial de la tierra, sobre una superficie de aproximadamente $50 \mathrm{~m}^{2}$, con cuchara estéril, hasta obtener un pool de unos 200-250 g de suelo mediante 5 a 7 recolecciones en distintos puntos de cada superficie estipulada. Las tierras colectadas se colocaron en sobres de papel estériles y se conservaron a $4^{\circ} \mathrm{C}$ hasta el momento de su procesamiento.

\section{Aislamiento fúngico técnicas: \\ Para el aislamiento de geohongos se utilizaron 2}

A) La técnica del anzuelo de Vanbreuseghem (3), empleando como fuente de queratina pelos de niños. Las muestras de tierra se dispusieron (por duplicado) en placas de Petri de $10 \mathrm{~cm}$ de diámetro, sobre ellas se colocaron fragmentos de 7 a $15 \mathrm{~mm}$ de pelos estériles. Luego se humedecieron con agua destilada con $0,25 \mathrm{~g} / \mathrm{L}$ de cloranfenicol y $0,5 \mathrm{~g} / \mathrm{L}$ de actidione. Se incubaron a temperatura ambiente $\left(25-28^{\circ} \mathrm{C}\right)$ durante 60 días. Cuando fue necesario, la tierra se rehumedeció con agua destilada. Las observaciones se realizaron cada 10 días.

B) Técnica de la dilución. Se pesó 1 gramo de cada muestra y se diluyó en $10 \mathrm{~mL}$ de agua estéril. De esta solución con concentración 1:10 se realizó otra 1.1000. De cada dilución se tomaron $0,2 \mathrm{~mL}$ que fueron dispensados, por duplicado, en placas de Petri de $10 \mathrm{~cm}$ de diámetro conteniendo agar papa dextrosa (PDA), adicionado con cloranfenicol $(0,25 \mathrm{~g} / \mathrm{L})$. La incubación se realizó a $25-28^{\circ} \mathrm{C}$ en estufa hasta 21 días. Las observaciones se realizaron semanalmente.

En ambos casos, los hongos que no pudieron ser clasificados con la observación macro y microscópica desde la placa original, fueron transferidos a medios de cultivos específicos para su identificación definitiva. En los casos que fue necesario, se emplearon técnicas y medios de cultivo especiales para identificar las especies. 
Algunos microhongos geofílicos en las planicies semiáridas del noroeste de la provincia de San Luis - Mangiaterra, et al.

Tabla 1.- Hongos aislados según la técnica del anzuelo queratínico: en muestreos de invierno (I) y verano (V), en distintos puntos de muestreo (1-10)

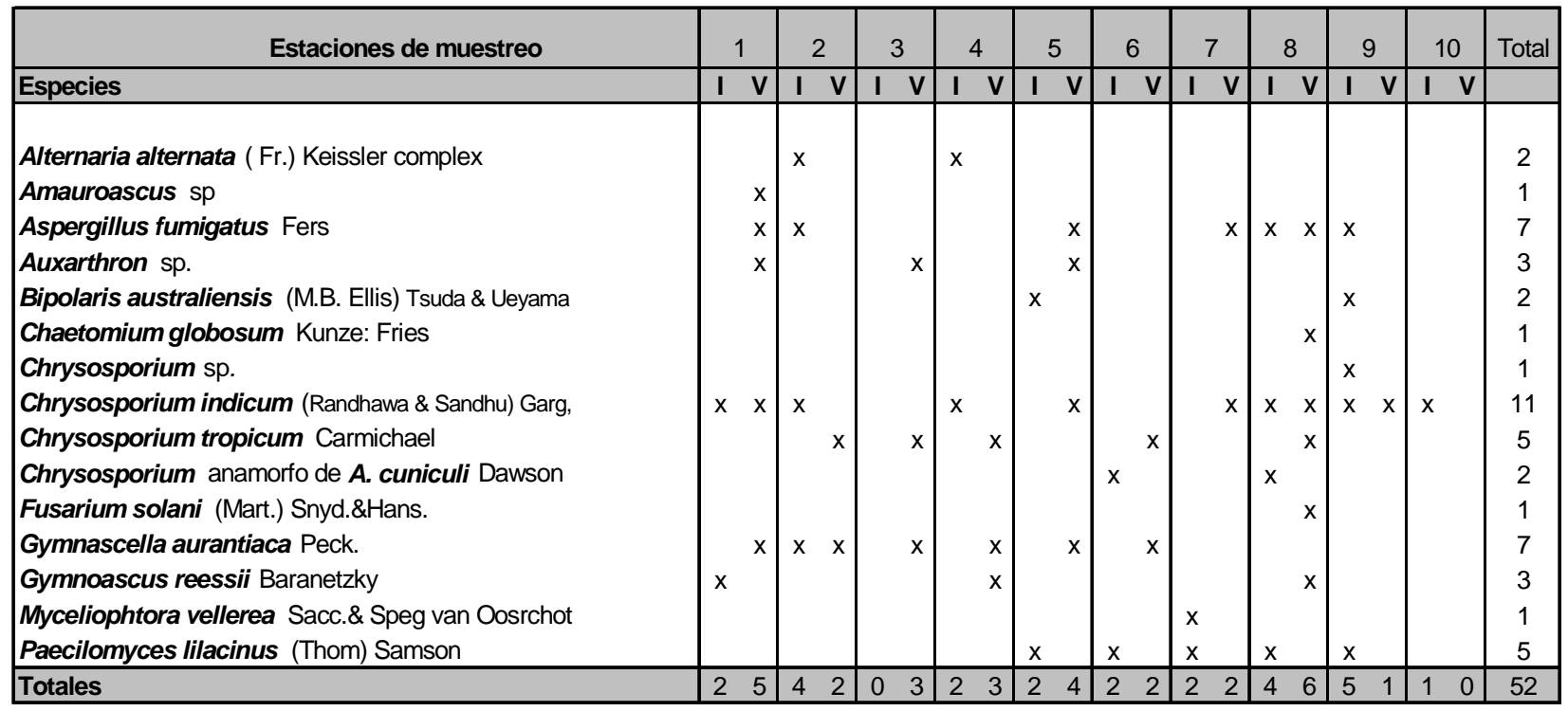

Cada taxa se contabilizó una sola vez en cada muestra, no importando si se repetía en la misma placa o en el duplicado. La frecuencia de cada género se calculó en base a la presencia de éstos en el total de las muestras $(n=20)$.

Cuando no se obtuvo desarrollo o escasos aislamientos, las muestras fueron procesadas de la misma manera hasta 3 veces.

Para la clasificación general de los hongos se utilizaron las siguientes referencias, Domsch et al. (1980); Currah (1985,1988); Von Arx (1981); Cano \& Guarro (1990); Sivanesan (1987); Gams (1971) (4,5,6,7,8,9).

\section{RESULTADOS Y DISCUSION}

Mediante las 20 muestras totales tomadas en invierno y verano con la técnica del anzuelo queratínico se aislaron 12 géneros y 15 especies fúngicas, mientras en las 20 procesadas por dilución en PDA se aislaron 15 géneros y 29 especies (Tablas 1 y 2).

Considerando las diferentes altitudes donde se realizó el muestreo, no se observaron diferencias considerables entre los asilamientos con ambas técnicas, ya que se repitieron los géneros encontrados entre los 500 y $1200 \mathrm{~m}$ sobre el nivel del mar.

A) Técnica del anzuelo queratínico. La mayoría de las especies aisladas pertenecieron a los Eurotiomycetes (Onygenales) representando el 65,4\% del total y 6 especies presentaron propiedades queratinolíticas (Onygenaceae y Arthrodermataceae) y 9 queratinofílicas. Dentro de las Onygenaceae queratinolíticas la frecuencia más alta corres- pondió a $\boldsymbol{C h}$. indicum, Ch. tropicum y Gymnascella aurantiaca (= Gymnoascus aurantiacus sensu Sole et al.(19),Gymnoascaceae), las restantes especies fueron esporádicas (Tabla 1). Los otros taxa con mayor frecuencia pertenecen a los Eurotiales: Aspergillus fumigatus y Paecilomyces lilacinus, el primero común en ambas estaciones y el segudo sólo en invierno, representando un 23\% del total (Tabla 1).

Los Onygenales tuvieron la mayor presencia especialmente en verano (Chrysosporium tropicum, Gymnascella aurantiaca y Ausxarthron sp.) y los lugares de muestreo con menor riqueza de especies fueron el tercer y el décimo, especialmente en invierno. La familia más representativa dentro del órden fue la Onygenacea con un $44,2 \%$ del total (Tabla 1 ).

Una de las razones que favorece la presencia de hongos querationofílicos - líticos, es la disponibilidad de queratina, que guarda gran relación con la existencia de animales $y / o$ humanos (10). Este estudio realizado en un área identificada como «travesía», es decir, de circulación y no de asentamiento por las condiciones de aridez y la carencia de agua potable (2), presenta una baja densidad poblacional humana y animal, tanto doméstica como silvestre. A estas condiciones podría atribuirse el predomino de integrantes del género Chrysosporium y la ausencia de representantes del género Microsporum y Trichophyton, ambos comunes en suelos preferentemente cultivados o abundantes en humus, pero también en los áridos (4). La metodología empleada (sólo 2 muestreos en el año) y las condiciones del sector, podrían explicar la baja presencia de algunos taxa queratinolíticos, sin embargo, la diversidad de géneros fue relativamente alta 


\begin{tabular}{|c|c|c|c|c|c|c|c|c|c|c|c|c|c|c|c|c|c|c|c|c|c|}
\hline \multirow{2}{*}{$\begin{array}{l}\text { Estaciones de muestreq } \\
\text { Especies y categorías fúngicas }\end{array}$} & \multicolumn{2}{|c|}{1} & \multicolumn{2}{|c|}{2} & \multicolumn{2}{|c|}{3} & \multicolumn{2}{|c|}{4} & \multicolumn{2}{|c|}{5} & \multicolumn{2}{|c|}{6} & \multicolumn{2}{|c|}{7} & \multicolumn{2}{|c|}{8} & \multicolumn{2}{|c|}{9} & \multicolumn{2}{|c|}{10} & \multirow[t]{2}{*}{ Total } \\
\hline & I & V & I & V & I & V & I & V & I & V & I & V & I & V & I & V & I & V & I & V & \\
\hline $\begin{array}{l}\text { Acremonium sp. } \\
\text { Acremonium strictum W. Gams } \\
\text { Acremonium minutisporum (Sukap.\& Thirum.) Gams } \\
\text { Alternaria alternata ( Fr.) Keissler complex } \\
\text { Aspergillus flavus Link } \\
\text { Aspergillus fumigatus Fres. } \\
\text { Aspergillus niger van Tieghem } \\
\text { Aspergillus sydowi (Bain. \& Sart) Thom \& Church } \\
\text { Aspergillus terreus Thom } \\
\text { Aspergillus versicolor (Vuill.) Tiraboschi } \\
\text { Bipolaris australiensis (Ellis) Tsuda \& Ueda } \\
\text { Chaetomium globosum Kunze: Fries } \\
\text { Chaetomium virescens (V.Arx) Udagawa } \\
\text { Cladosporium cladosporioides (Fres.) de Vries } \\
\text { Cunninghamella elegans Lendner } \\
\text { Cunvularia lunata (Wakker)Boedjin } \\
\text { Emericella nidulans (Eidam) Wint. } \\
\text { Fusarium solani (Mart.) Sacc. } \\
\text { Fusarium chlamydosporum Wollenw. \& Reinking } \\
\text { Micelio dematiáceo toruloide } \\
\text { Micelio hialino sin fructificar } \\
\text { Paecylomyces lilacinus (Thom) Samson } \\
\text { Penicillium spp. } \\
\text { Penicillium aculeatum Raper \& Fennell } \\
\text { Penicillium pinophilum Hedgcock } \\
\text { Penicillium simplicissimum (Oud.)Thom } \\
\text { Picnidios dematiáceos con setas } \\
\text { Rhizopus oryzae Went \& Prinsen Geerlings } \\
\text { Rhizopus stolonifer (Ehrenb.) Vuill. } \\
\text { Ulocladium atrum Preuss } \\
\text { Trichoderma harzianum Rifai } \\
\text { Trichoderma sec. longibrachiatum Rifai } \\
\end{array}$ & $\begin{array}{l}X \\
X\end{array}$ & $x$ & $x$ & $\begin{array}{l}x \\
x\end{array}$ & X & $\begin{array}{l}x \\
x\end{array}$ & $\begin{array}{l}X \\
X\end{array}$ & $x$ & $x$ & $x$ & $\begin{array}{l}x \\
x\end{array}$ & 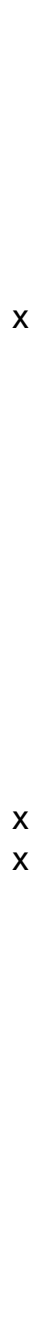 & $x$ & $\begin{array}{l}x \\
x\end{array}$ & $\begin{array}{l}x \\
x\end{array}$ & $x$ & $x$ & $\begin{array}{l}x \\
x \\
x\end{array}$ & & $x$ & $\begin{array}{c}2 \\
2 \\
1 \\
6 \\
6 \\
7 \\
7 \\
6 \\
7 \\
3 \\
1 \\
2 \\
1 \\
7 \\
1 \\
2 \\
2 \\
2 \\
1 \\
4 \\
12 \\
4 \\
2 \\
1 \\
4 \\
1 \\
1 \\
2 \\
2 \\
1 \\
5 \\
1\end{array}$ \\
\hline Totales & 6 & 7 & 7 & 4 & 6 & 3 & 6 & 2 & 6 & 2 & 10 & 3 & 3 & 5 & 9 & 7 & 13 & 6 & 0 & 1 & 106 \\
\hline
\end{tabular}


en este grupo a pesar de la competencia por el escaso substrato queratínico, lo que indica la capacidad de sobrevivencia, adaptación y dispersión de los Onygenales y taxa relacionados en estos habitat adversos. Destaca Ch. indicum por su distribución cosmopolita en muchos tipos de suelo y localizaciones geográficas incluyendo Argentina $(13,14,15,16)$.

\section{B) Técnica de dilución del suelo.}

En las diluciones, la mayoría de la presencia fúngica correspondió a hongos mitospóricos y 3 meiospóricos (> $90 \%$ ) con un 25\% de ellos con micelio dematiáceo, mientras la presencia de integrantes de Zygomycota fue de sólo 3 taxa (Tabla 2). La riqueza de especies fue levemente mayor en verano que en invierno y los lugares de muestreo con mayor abundancia fueron el 8 y 9 en ambas estaciones y el menor el 10 en verano (Tabla 2). Algunas especies se presentaron únicamente en invierno como Alternaria alternata, Aspergillus niger y los integrantes del género Trichoderma y en verano fueron principalmente los integrantes del género Acremonium; ninguno de los Onygenales fueron aislados con esta metodología.

Los géneros con mayor diversidad de especies fueron: Aspergillus (7) con un 35,8\% del total de la presencia, micelio hialino sin fructificar con un $11,3 \%$ y Penicillium (más de 4) con un 8,5\%. Mientras la categoría y las especies de mayor constancia en el tiempo (en 5 o más zonas de aislamiento) fueron en órden decreciente: micelio hialino sin fructificar, A. fumigatus, A. niger, A.terreus, C. cladosporioides, A. flavus, Alternaria alternata y Trichoderma harzianum. Los otros géneros sólo se presentaron 1 a 3 veces en las estaciones de muestreo (Tabla 2).

La mayoría de los hongos aislados son saprófitos del suelo y restos vegetales, con características geno $\mathrm{y}$ fenotípicas que les permiten adaptarse a diferentes categorías econutricionales. Los cuadros alérgicos y las infecciones oportunistas producidas por las especies de hongos mitospóricos integrantes de los Ascomycota aislados, han sido descritas para varios representantes de los géneros Acremonium, Aspergillus, Bipolaris, Curvularia, Fusarium, Rhizopus y Trichoderma en muchos lugares del mundo (11). Cladosporium es un reconocido aeroalergeno y también ha sido encontrado como patógeno oportunista en infecciones pulmonares, cutáneas y oculares (11). Los miembros del género Aspergillus se encuentran ampliamente distribuidos en la naturaleza, especialmente en las zonas cálidas (12) y tienen dos características particulares: la termotolerancia y la xerofilia. Destaca A.fumigatus, que tiene un óptimo de crecimiento a los $45^{\circ} \mathrm{C}$ y puede desarrollarse con un mínimo de aw de 0,85 (17).

A pesar que ambas técnicas de cultivo no son comparables en el aislamiento de hongos geofílicos, la presencia de A. fumigatus y Paecilomyces lilacinus fue bastante semejante, mostrando que ambas metodologías presentan buena sensibilidad para estos taxa. El primero, puede asociarse con una amplia variedad de cuadros clínicos que van desde el aspergiloma a la aspergilosis invasiva, pasando por varios tipos de manifestaciones alérgicas. El segundo, también de amplia distribución en climas cálidos y por su capacidad de crecer a $37^{\circ} \mathrm{C}$, puede producir varios cuadros clínicos oportunistas, como: sinusitis en pacientes diabéticos, infecciones cutáneas, oculares y onicomicosis (18).

\section{REFERENCIAS}

1.- Hawksworth, D.L. \& Ritchie J.M. (1993). Biodiversity and Biosistematic priorities: microorganisms and invertebrates. C.A.B. International. Wallingford UK.

2.- Chiozza E, Figueira R. (1981). Provincia de San Luis. En Atlas físico de la República Argentina. Centro Editor de América Latina. Vol 1.pp. 69-71

3.- Vanbreuseghem, R. (1952). Technique biologique pour l'isolement des dermatophytes du sol. Ann. Soc. Belge de Med. Trop. 32:173-178

4.- Domsch, K.H.; Gams, W. \& Anderson, T. (1980). Compendium of soil fungi.Vol 1. Acad. Press. London.

5.- Currah, R.S. (1985). Taxonomy of the Onygenales, Arthrodermataceae, Gymnoascaceae, Myxotrichaceae, Onygenaceae. Mycotaxon 24:1-216.

6.- Von-Arx, J.A. (1981). The genera of fungi sporulating in pure culture. $3^{\mathrm{a}}$ ed. Edit J. Crame. Vaduz.

7.- Cano, J. \& Guarro, J. (1990). The genus Aphanoascus. Mycol. Res. 94: 355-337.

8.- Sivanesan, A. (1987). Graminicolous species of Bipolaris, Curvularia, Drechslera, Exserohilum and their teleomorphs. Mycological papers, $N^{\circ}$ 158. C.A.B. International Mycological Institute.

9.- Gams, W. (1971). Cephalosporium-artige Schimmelpilze (Hyphomycetes), G Fisher, Stuttgart.

10.- Abril, M.J.; Guisantes, J.A. \& Rubio, M.F. (1991). Estudio de los dermatofitos y otros hongos queratinofílicos de los suelos de Navarra. Rev. Iberoam Micol 8:79-88

11.- Hoog, G.S.; Guarro, J.; Gené, J. \& Figueras, M.J. (2000). Atlas of Clinical Fungi. CBS. and Universitat Rovira I Virgili.

12.- Klich, M.A. (2002). Biogeography Aspergillus species in soil and litter. Mycologia 94:21-27

13.- Deshmukh, S.K.; Agraval,S.C. \& Jaun, P.C. (2000). Isolation of Dermatophytes and other keratinophilic fungi from soils of Mysore (India). Mycoses 43:55-57 
Algunos microhongos geofílicos en las planicies semiáridas del noroeste de la provincia de San Luis - Mangiaterra, et al.

14.- Abdel-Fattah, H.M.; Moubasher, A.H. \& Maghazy, S.M. (1982). Keratinophilic fungi in Egyptian soils. Mycopathologia 79:49-53

15.- Mangiaterra, M.; Giusiano, G.; Deluca, G.\& Alonso, J.M. (2000). Geohongos queratinofílicos en áreas de recreación de jardines de infantes en Resistencia (Argentina). Boletín Micológico $15: 101-106$

16.- Piontelli,E. \& Caretta, G. (1974). Considerazioni ecologiche su alcuni geomiceti isolati su substrati cheratinici in localitá montagnose delle Ande del Chile. Rev. Patol. Vegetale 10:261-314

17.- Frisvad, J.C. \& Samson, R.A. (1991). Filamentous fungi in foods and feeds:ecology, spoilage and mycotoxin production. In Handbook of Applied Mycology. Vol. 3. Foods and Feeds. eds. Arora, D.K., Mukerji, K.G. \& Marth, E.H. New York:Marcel Dekker, pp. 31-68
18.- Williamson,P.;Kwon-Chung, K.J. \& Gallin, J.I. (1992). Successful treatment of Paecilomyces varioti infections in a patient with chronic granulomatous disease an a review of Paecilomyces species infections. Clin. Infect. Dis. 14:1023-1026

19.- Sole, M.; Cano, J.; Pitarch, L.; Stchigel, A.M.; Guarro,J. (2002). Molecular phylogeny of Gymnoascus and related genera. Studies in Mycology 47:141-152 